\title{
REFLEXIONES SOBRE LA INVESTIGACIÓN CONTABLE EN COLOMBIA
}

Carlos Eduardo Castaño-Rios 
Panorama Económico, 24 (Octubre 2016 - Septiembre 2017), pp. 163-174

Carlos Eduardo Castaño-Rios

\title{
Reflexiones sobre la investigación contable en Colombia
}

\begin{abstract}
Resumen
La investigación contable colombiana ha sido un tema de interés desde sus inicios en la década de los años 70 y hoy la gran inquietud que surge es sobre el impacto que ha tenido el desarrollo en una comunidad de investigación y en el contexto internacional. Un barrido por las distintas publicaciones nacionales que aglutinan una buena parte de la producción de investigadores colombianos en contabilidad, deja en evidencia que aún existen limitaciones que no han permitido que se consolide como la ausencia de debate entre colegas, la falta de apoyo institucional, la poca cercanía con la realidad empresarial, entre otras. Bajo ese panorama, se proponen algunas ideas de acercamiento para trabajo en red y fortalecimiento de capacidades en investigación, que de llevarse a cabo, podrían potenciar de manera importante al país, en un escenario de visibilidad internacional.
\end{abstract}

Palabras Clave: Investigación contable colombiana, comunidad de investigación.

Clasificación JEL: M40, M41, M42, M49, I23.

\section{Reflections on accounting research in Colombia}

\begin{abstract}
Colombian accounting research has been an area of interest since its inception in the 1970s; however, the great concern that arises nowadays is the impact of development on a research community and on the international context. A sweep of the different national publications that gather together a large part of the Colombian researchers' production in accounting, showing that there are still limitations such as the absence of debate among colleagues, lack of institutional support, very little closeness to business reality, among others; which have not allowed a consolidation. Under this scenario, this article addresses some ideas on how to approach for networking and strengthening research capacity, which, if carried out, could significantly enhance the country in terms of international visibility.

Keywords: Colombian accounting research, research network.

JEL Classification: M40, M41, M42, M49, I23.
\end{abstract}

\section{Réflexions sur la recherche comptable en Colombie}

\begin{abstract}
Résumé
La recherche comptable colombienne est un domaine d'intérêt depuis sa création dans les années 70. Actuellement une inquiétude qui émerge concernant l'impact du développement dans une communauté de recherche et le contexte international. Un échantillonnage des différentes publications nationales qui collectent une grande partie de la production des chercheurs colombiens en comptabilité montre qu'il existe encore des obstacles nuisant à une correcte consolidation, tels que l'absence d'échange entre collègues, le manque de soutien institutionnel, la distanciation avec la réalité entrepreneuriale, parmi tant d'autres. Dans ce sens, quelques idées sont proposées sur la façon d'aborder le travail en réseau et le renforcement des capacités de recherche. Leur implémentation pourrait améliorer considérablement la visibilité internationale du pays.
\end{abstract}

Mots-clés: Recherche comptable colombienne, communauté de recherche.

Nomenclature JEL: M40, M41, M42, M49, I23. 


\section{REFLEXIONES SOBRE LA INVESTIGACIÓN CONTABLE EN COLOMBIA}

"A menudo se acepta la idea de una investigación con cierto aire de exclusividad, un reto intelectual asumido por una élite dotada para desarrollarla; sin embargo, no se puede perder de vista que la investigación tiene una naturaleza inherente al ser humano y, por lo tanto, no debería constituirse en un atributo exclusivo para algunas personas sino un compromiso colectivo con el devenir de la humanidad"

Machado y Patiño, 2016, p. 51.

\section{INTRODUCCIÓN}

Este trabajo surge como respuesta a un interés común de la comunidad investigativa colombiana, que requiere estar en permanente evaluación por parte de sus interlocutores a fin de proponer nuevas alternativas de mejoramiento continuo. También nace con el propósito de describir la realidad actual de la investigación contable del país y así contribuir con algunos aportes sobre lo que podría ser un mejor futuro para la academia contable colombiana.

Para lograr lo propuesto, se realizó una revisión general sobre las principales revistas contables colombianas y se complementó con la lectura de memorias de algunos eventos de investigación contable en el país como el Simposio Contaduría Universidad de Antioquia, Encuentro internacional de Perspectivas Críticas de la contabilidad contemporánea, Simposio Nacional de Investigación y Docencia Contable, Encuentro nacional de profesores de contaduría pública, tomando como principal período de evaluación las publicaciones de los últimos cinco años, pero sin desconocer el contexto histórico en el cual se ha desarrollado la investigación contable en Colombia.

\footnotetext{
${ }^{1}$ Autor para correspondencia. Correo electrónico: eduardo.castano@udea.edu.co
} 
En este orden de ideas, el texto presenta inicialmente lo que ha significado la investigación contable en Colombia y las principales limitaciones que ha enfrentado. Posteriormente, se evalúa, en términos descriptivos, el impacto de estos procesos de investigación en los ámbitos de formación del contador público y de la práctica profesional. Más adelante, se enuncian alternativas de solución a los problemas de la investigación contable en el país e inclusive se proponen temáticas que podrían ser abordadas en el corto plazo. Para finalizar, se realiza una breve reflexión sobre el futuro de la investigación contable en el país.

\section{CONTEXTO DE LA INVESTIGACIÓN CONTABLE COLOMBIANA EN LA ACTUALIDAD}

Tras casi dos décadas transcurridas del siglo XXI, es posible afirmar, sin temor a equivocarse, que en la academia contable colombiana se abordan tantas o más problemáticas de investigación por cuantos investigadores estén sumando trabajos para la disciplina. Un breve repaso por revistas contables colombianas como Contaduría Universidad de Antioquia, Cuadernos de contabilidad, Lúmina, Visión Contable, Colombian Accounting Journal, Activos, revista colombiana de contabilidad (antes revista ASFACOP), revista legis de contabilidad y auditoría, Adversia y algunas otras revistas interdisciplinarias (que acogen a la contabilidad como una de las áreas del conocimiento aceptadas para su publicación) como Innovar, Cuadernos de administración, Porik An, Science of Human Action, Panorama Económico, Criterio Libre, Revista investigación y reflexión de la Universidad Militar Nueva Granada, Ciencias estratégicas, Teuken Bidikay, En-contexto, permite reconocer esta diversidad temática y problemática con trabajos desarrollados sobre la revelación de la información financiera por internet, la enseñanza de la contaduría pública, los efectos de la implementación de normas internacionales de información financiera (NIIF) en Colombia, la contabilidad de gestión y de costos aplicables en sectores o tipos de entidades particulares, discusiones sobre la forma en que se hace investigación y que dejan sobre la mesa nuevas alternativas para llevar a cabo la investigación desde corrientes heterodoxas del conocimiento, entre otros.

No obstante, es evidente la desconexión entrecolegasacadémicosdela contabilidad. Son pocos los trabajos que se observan publicados que integren profesores de dos o más instituciones, donde se compartan esfuerzos investigativos; y esta situación se profundiza, cuando se observa con mayor detenimiento la ausencia de procesos de lectura y revisión de trabajo entre los mismos colegas colombianos, pues es de resaltar que la mayoría usa referentes extranjeros o ajenos a la profesión, considerándolos quizás un mejor soporte para sus trabajos. Bajo este contexto, es notable la limitación para la retroalimentación entre los trabajos que abordan un área del conocimiento común (Macías, Alzate, Bautista y otros, 2014).

Adicionalmente, parece natural, en el contexto colombiano, la constante migración académica de los investigadores contables. Esto se constata cuando es evidente que un mismo autor pasa de un tema a otro y en la mayoría de los casos, deja abandonados sus temáticas anteriores, lo que parece fuese su última palabra al respecto. Además, es posible que cada lectura que se realiza de un autor colombiano contable a partir de un tema, se convierta en un eterno retorno ${ }^{1}$

1 Metafóricamente aludiendo al libro El eterno Adán de Julio Verne. 
al inicio desde cero en un mismo aspecto que quizás ya fue abordado por otro investigador, pero que aparece como un desconocido para este nuevo escrito. Sobre este asunto, se puede tomar como evidencia el trabajo plasmado en el libro de Huellas y devenir contable (Machado, 2012), en el cual, se puede observar a través de sus páginas este fenómeno de diversos autores que migran de línea de investigación constantemente, pero que en el fondo, no logran consolidarse con liderazgo en ninguna de ellas.

Por su parte, en relación a los perfiles propios de las corrientes que dominan el pensamiento para la construcción de conocimiento contable, se encuentra que la participación en los diversos eventos sobre contabilidad en el país y la lectura de las revistas contables e interdisciplinarias anteriormente mencionadas, llevaría a plantear hipotéticamente que la corriente de investigación que prima en la academia contable es la ortodoxa (tradicional de corte positivista), lo que conllevaría a la existencia de múltiples trabajos orientados por el método científico y enfocados hacia aspectos pragmáticos y observables en una realidad de estructura concreta susceptible de ser representada tal cual como se presenta (Ryan, Scapens, Theobald, 2004). Al contrario, es más probable evidenciar que las publicaciones y conferencias en eventos, se centran en miradas más pluralistas y abiertas, con un enfoque alternativo, interpretativo o crítico, pero alejado de academias como la americana o mexicana que le apuestan al análisis cuantitativo y uso de herramientas econométricas como la medida de la verdad en contabilidad. De este modo, las publicaciones que se pueden evidenciar de los contadores públicos colombianos que están inmersos en la academia centran su atención a las reflexiones, análisis documental, y en algunas ocasiones, a estudios de caso (Gómez, 2009).

\section{PRINCIPALES LIMITACIONES PARA CONSOLIDAR LA INVESTIGACIÓN CONTABLE EN COLOMBIA}

Como se ha venido esbozando, la investigación contable colombiana ha presentado limitaciones de diversas índoles en su progreso que datan desde sus inicios en la década de los 70s y que aunque se han reducido gracias a la dedicación en investigación para el mejoramiento de la calidad de los programas de contaduría pública, aun no se ha consolidado. A propósito de esta situación, vale la pena considerar algunas de esas restricciones que actualmente viven la mayoría de los investigadores contables en el país:

- Uno de los grandes problemas que afronta la comunidad investigativa colombiana está relacionado con la pluralidad temática y la ausencia de debate entre colegas internamente. En este sentido, por cada investigador aparecen una o varias líneas de investigación que a renglón seguido desaparecen o se reinventan cuando el mismo interés de ese investigador se ve permeado por la necesidad de abordar nuevas temáticas.

- Pocos incentivos para la investigación contable. En la actualidad, la investigación contable ha recaído principalmente sobre los académicos contables del país, quienes en su gran mayoría, realizan grandes esfuerzos para lograr proyectos y documentos derivados de los mismos, recibiendo a cambio quizá, sólo la satisfacción personal por su aporteal conocimiento. Se debe denotar que la mayoría de investigadores del área contable no tienen una dedicación completa 
a esta actividad sino que deben realizar sus funciones de docencia, apoyo administrativo, extensión, para apoyar en el logro de las metas propuestas desde la administración de los programas. Se evidencia a lo largo y ancho del país, que existen algunos beneficios como bonificaciones, puntos salariales, descargas de tiempo directo de docencia, que aunque no pueden ser el centro de atención de los investigadores, porque la investigación debe ser por convicción y con un fin común para lograr el mejoramiento de la calidad de vida humana, se quedan cortos ante el esfuerzo que es necesario realizar para realizar estas actividades. En pocas palabras, sería interesanteque los mismos programas de contaduría pública le den valor a sus profesores investigadores retribuyendo su actividad al destacarlos frente a la comunidad académica, brindándoles tiempo para la investigación (sin desconocer su capacidad docente), generando espacios para compartir sus resultados, apoyando la formulación de nuevos proyectos y la publicación de sus esfuerzos.

- La ausencia de programas de investigación de largo plazo (Rueda, 2009). La necesidad permanente de las instituciones educativas por presentar resultados, en pro del cumplimiento de los distintos indicadores de calidad e impacto en la investigación, hacen que los investigadores del área contable tengan que formular proyectos de uno o máximo dos años, y a partir de estos presentar resultados rápidamente para eventos académicos y revistas, principalmente indexadas, para cumplir con las metas de la institución en cuanto a indicadores. Esta situación, lleva a que gran cantidad de estas las investigaciones carezcan de un importante interés por aportar un avance a la disciplina y se ciñan simplemente a la recurrente repetición de lo que otros ya han hecho.

- Las limitaciones metodológicas para formular proyectos que puedan ser aprobados para obtener recursos de instituciones externas. En este sentido, el profesor Rueda (2007) expresa que "se han generado serias presiones para que en las universidades los profesores de planta formulen proyectos de investigación, y en muchos casos, ellos carecen de habilidades, conocimientos y estructuras que les permitan realizar esta labor. Los proyectos se entienden como una serie de actividades que hay que "HACER"” (p. 121).

- Ausencia de integración entre programas de pregrado y posgrado en investigación contable. En muchas instituciones educativas donde se ofrece el programa de contaduría pública, es común encontrar ofertas de formación posgraduales, en especial, de especialización; no obstante, cuando el estudiante de posgrado desea realizar su trabajo de monografía para cumplir con este requisito de graduación tiene dificultades para plantear problemas y sugerir caminos de solución a partir de proyectos que efectivamente aporten algún valor agregado una vez ejecutado el trabajo de campo. La situación es mucho más compleja si se piensa en programas de maestría o doctorado, para los cuales, estos profesionales no llegan con la más mínima idea para aportar en la búsqueda de nuevos conocimientos. Esta falta de articulación también ha llevado a que los contadores públicos colombianos tengan poco interés en realizar maestrías y doctorados.

- La investigación contable colombiana aparece con una fuerte limitación en la 
búsqueda de la solución de problemas de la realidad, pues no es un secreto que a la fecha, las empresas no reconozcan que su contador público pueda además de sus funciones esperadas, desarrollar investigación al interior de la organización como respuesta a problemas latentes relacionados con el saber contable.

- La falta de redes de investigación que operen en cooperación. Si bien es cierto que con el pasar de los días son más los convenios, acuerdos y redes en las que aparecen distintas instituciones de educación superior en contaduría pública, esto no ha conllevado a procesos investigativos aunados e integrados entre estas mismas, pues aspectos como la ausencia de profesores investigadores, las múltiples cargas que se suman a la docencia y el aumento de la cobertura, han hecho que estos documentos queden en los anaqueles de las entidades firmantes y que los resultados tangibles de las redes no se logren ver en la comunidad académica.

Estos tipos de limitaciones enunciadas se ven reflejadas en la calidad de los programas de contaduría pública, la poca integración que se ha logrado entre los procesos de investigación y los modelos de formación, la casi nula relación entre la investigación contable y los problemas reales de las organizaciones. Además, la mayoría de profesionales del área contable no se visualiza a futuro con habilidades para la investigación, es inclusive, una persona que sólo se acerca nuevamente a la academia pero con la finalidad de recibir formación para el trabajo, en el hacer, evitándose la aventura del pensar para mejorar.

Tal como lo expresa el profesor Rojas (2009), con respecto a los procesos de formación en investigación:
Creemos que si los estudiantes no pueden orientarse con una mínima suficiencia en la formulación de un proyecto de investigación en contabilidad financiera, contabilidad pública, o en contabilidad ambiental, es porque los programas de estudio no lograron cultivar un pensamiento crítico que los incluya en la sociedad como re-constructores de su mundo (p. 203).

Así las cosas, vale la pena realizar un breve análisis sobre los impactos que ha tenido la producción investigativa contable en el país.

\section{A PROPÓSITO DEL IMPACTO DE LA INVESTIGACIÓN CONTABLE COLOMBIANA}

En términos del impacto que ha logrado la investigación contable colombiana, es válido hacer dos grandes distinciones. La primera, en torno a su impacto en la misma formación de los contadores públicos. La segunda, frente a los resultados de investigación y su impacto en la práctica profesional del contador público.

Frente a los procesos formativos, vale la pena resaltar que en la actualidad algunos programas de contaduría pública del país incluyen dentro de sus currículos como parte de la formación obligatoria del contador público, contenidos de investigación y teoría contable. No obstante no es algo generalizable pues en muchas ocasiones se confunde la misma formación en investigación como una transmisión de conocimientos sobre las partes de un proyecto sin que el estudiante tenga la necesidad de pensar en los problemas propios de su disciplina y cómo resolverlos a través de la investigación. También, se considera valioso que algunos programas de contaduría pública incluyan dentro de sus opciones de grado, la elaboración de un trabajo de grado, implicando con ello a la investiga- 
ción, sin embargo, la mayoría de estudiantes optan por hacer una práctica, pasantía u otra modalidad, antes de iniciar un trabajo de grado cuando este es opcional. En el caso de la Universidad de Antioquia, es notable el esfuerzo del Departamento de Ciencias Contables por consolidar una propuesta curricular, en la cual, sus estudiantes tienen como requisito obligatorio para culminar los estudios profesionales, diseñar y ejecutar un trabajo de grado, teniendo como única alternativa de cambio de esta modalidad, la participación del estudiante en un proyecto de investigación como estudiante en formación (Carvalho y otros, 2006).

Al respecto el profesor Rueda (2007) manifiesta que "existe poca articulación entre las asignaturas que abordan la teoría, la investigación, la epistemología o algunos marcos conceptuales, y las denominadas, asignaturas "técnicas"”' (p. 122).

En cuanto a los procesos de investigación para la formación de pregrado, en particular, el caso del curso de metodología de la investigación contable de la Universidad del Valle (sede Norte del Cauca), el profesor Grajales (2010) expresa, sobre el proceso de escritura de anteproyectos de investigación, que "dicha actividad escritural se dificulta debido a que la mayoría de estudiantes no le gusta leer y/o leen muy poco" (p. 146), aludiendo a este curso que está programado para estudiantes de último semestre de contaduría pública en esta universidad y en el cual se parte del objetivo general "facilitar la formulación de un anteproyecto de investigación" (p. 148). De este modo, el autor también refiere a la necesidad de articulación de conocimientos básicos de lectura y escritura para afrontar la construcción de una propuesta investigativa.
Asímismo, en relación al proceso formativo, es notable el incremento de eventos para la participación de estudiantes, con un importante auge en la primera década del siglo XXI en adelante. Este tipo de eventos solicita ponencias y trabajos escritos por los estudiantes, derivados de sus procesos de reflexión y de su participación en proyectos de investigación. Con esta modalidad aparecen diversos Congresos Nacionales de estudiantes de contaduría pública como los de la Pontificia Universidad Javeriana, Universidad Cooperativa de Colombia, Universidad Santo Tomás, Federación Nacional de estudiantes de contaduría pública ${ }^{2}$-FENECOP-, aunque para este año aún no se había creado FENECOP), Universidad de la Salle, entre otras, asimismo, se encuentra el Encuentro Nacional e Iberoamericano ${ }^{3}$ de Ensayo Contable, liderado por la Universidad de Antioquia, donde se evidencian grandes esfuerzos para mejorar la calidad de la escritura de los estudiantes de contaduría pública con un fuerte énfasis en la construcción conjunta y plural de conocimiento a partir de las reflexiones que se realizan por parte de los estudiantes participantes.

\footnotetext{
2 Este Congreso de estudiantes tiene una gran tradición, el primero fue desarrollado en el año 1984 y para el año 2016, también se lleva a cabo junto con la VII versión del congreso dirigido para estudiantes de contaduría pública en Latinoamérica. A pesar de que el Congreso inició en 1984 en dicha fecha aún no se había creado FENECOP, tal como consta en la historia de los congresos que se puede consultar en la página http:// www.fenecop.org/quienes-somos.

3 En el año 2014 se llevó a cabo la primera versión extendiendo la invitación a participar de otros países, no obstante, los ensayos enviados desde otros países diferentes a Colombia no lograron pasar a la final del certamen. Las memorias con los ensayos clasificados se pueden encontrar en el portal de la revista virtual de estudiantes de contaduría pública Adversia, de la Universidad de Antioquia, específicamente en su número 15 de julio-diciembre 2014 (ver publicación en el link http://aprendeenlinea.udea.edu.co/revistas/ index.php/adversia/issue/current/showToc)
} 
En este mismo orden de ideas, se ha ampliado la visión interdisciplinaria de la contabilidad y es mucho más difundido el concepto de contabilidad como ciencia social, superando en gran medida, el tradicional esquema de una contabilidad técnica, ya esquematizada y "monótona", aludiendo a los grandes prejuicios que se le atribuyen a la contabilidad y en especial al profesional que ejerce como contador público (Grajales, 2007).

Otro aporte para la academia contable nacional ha sido el creciente número de semilleros de investigación, en el cual, aunque la participación de estudiantes todavía es limitada, sigue avanzando e integrando el conocimiento propio de la profesión, con los procesos investigativos.

No obstante estos logros mencionados sobre el impacto de la investigación en la formación de los contadores públicos del futuro, el efecto ha sido limitado, pues cuando se trata de reformar currículos en los programas de contaduría pública o ajustarse a las necesidades del mercado laboral, lo primero que se recorta (sin previo análisis académico) es la formación epistemológica y metodológica sobre contabilidad, llevándola a un segundo plano y ofreciéndola en muchos casos comoalternativa a estudiantes que pierden la motivación e interés por fortalecer las competencias que les puede brindar este tipo de conocimiento básico.

Entre tanto, el impacto de la investigación contable en relación a la práctica profesional ha sido casi nulo. Las organizaciones donde se desempeña el contador público no ven a este profesional como un generador de conocimiento $\mathrm{y}$ alternativas de solución de problemas a partir de procesos de investigación, excepto, en la misma actividad de docencia, donde es uno de los requisitos que hoy en día se viene imponiendo para el acceso a un lugar de trabajo de carrera. Pero ante el desalentador panorama, se puede expresar que el contador público que se forma en investigación está en capacidad de discernir sobre el efecto que produce su actuación sobre la misma organización y la sociedad en la cual está inmerso.

\section{¿QUÉ ALTERNATIVAS DE SOLUCIÓN SE OFRECEN?}

Considerando el panorama para la investigación contable en el país, se hace necesario proponer algunos caminos potenciales para trabajar por parte de las instituciones que hoy tienen a su cargo este tipo de procesos. A propósito de lo mencionado, sería bastante valioso que las distintas entidades que hoy agrupan programas de contaduría pública $\mathrm{y}$ contadores públicos que investigan, trabajen de forma mancomunada para continuar en una senda de fortalecimiento de la investigación como eje central de los futuros profesionales contables. A partir de esta idea, se ofrecen a la comunidad académica contable las siguientes alternativas, sin querer con esto indicar que lo planteado es una lista exhaustiva de las rutas a seguir:

- En primer lugar, se requiere que los mismos programas de contaduría pública enlacen la investigación en su visión. Esto implicaría favorecer los escenarios para el desarrollo permanente de investigación en sus programas, con profesores que tendrían la disponibilidad para presentar proyectos, participar de eventos académicos, poner sus ideas en debate, acompañar la formación investigativa de sus estudiantes de forma individual o a través de semilleros de investigación. 
- Fortalecimiento de los profesores en el conocimiento de la metodología de la investigación. Es importante que las instituciones realicen formación de su equipo docente en metodología de la investigación, pues son diversas las tipologías y métodos de investigación aplicables para generar nuevos conocimientos, pero la colonización del tradicional método de investigación científico ha limitado ese mismo potencial de avance de la contabilidad en tanto se le ubica en el campo de las ciencias sociales. Este mejoramiento y profundización de conocimientos en el estudio de los métodos para la investigación, se considera un reto importante para la comunidad académica contable del país (Ospina y Castaño, 2012).

- Creación de semilleros de investigación en contabilidad con escenarios de difusión interuniversitarios. Por ejemplo, un Encuentro Nacional de Semilleros de Investigación en Contabilidad. Esta propuesta surge al encontrar que los trabajos de investigación en contabilidad muchas veces se circunscriben en el mismo ámbitodelasingenierías odelas ciencias exactas, donde naturalmente será complejo encontrar un interlocutor del conocimiento para el fortalecimiento de la investigación en contabilidad.

- Diseño de programas de investigación de largo aliento, que no se limiten al resultado para llenar indicadores, sino que tengan un propósito básico para la solución de problemas tangibles relacionados con la disciplina contable.

- Mejoramiento de la relación Universidad- Empresa. Esto facilitaría el acceso a problemas tangibles en las organizaciones y al reconocimiento del contador público como agente que puede aportar soluciones desde el proceso investigativo.

\section{¿SOBRE QUÉ INVESTIGAR EN CONTA- BILIDAD EN EL FUTURO CERCANO?}

Aunque los temas de investigación dependen en gran medida del interés propio de los investigadores, vale la pena llamar la atención sobre algunos ámbitos relacionados con la disciplina contable que requieren de un abordaje desde la investigación en el corto plazo como son:

- La evaluación de los impactos reales de la implementación de las actuales normas de contabilidad en Colombia por subsectores de la economía y por tamaños empresariales, tanto en términos económicos como de calidad y de la utilidad de la información para los procesos de toma de decisiones organizacionales.

- Los efectos del reajuste en los modelos de auditoría aplicables en Colombia y los cambios en la institución del revisor fiscal una vez implementado el modelo de normas internacional de auditoría (NIA) en el país, pues es evidente que la naturaleza y objetivos de cada uno de estos modelos de control externo son diferentes.

- El debate sobre la formación del contador público en Colombia, son sólo de su formación básica en el pregrado, sino también de los procesos de certificación y acreditación de requisitos para ejercer la profesión en el país, así como la formación en posgrados.

- Los nuevos escenarios de reporte de información financiera integrada y su aplicación en el país.

- El comportamiento de los mercados accionarios una vez reportada información financiera bajo la nueva normativa contable implementada en Colombia.

- Las discusiones sobre la ética profesional en el uso o aplicación de criterios profesionales bajo el modelo de normas contables actuales basado en estándares internacionales. 
- El eterno dilema coyuntural en Colombia en relación a los tributos, pues está en camino una reforma estructural del estatuto tributario, pero, no se puede olvidar el latente escenario de posconflicto tras la firma del acuerdo del gobierno con las FARC y el inicio de negociaciones con el ELN.

Recientemente, una publicación del Departamento de Ciencias Contables, denominada Caminos Contables, ha dado algunas ideas sobre las problemáticas que giran en torno a las líneas de investigación contable que allí se promueven a saber: costos y gestión, contabilidad y finanzas, contabilidad y recursos públicos, análisis de los procesos tributarios, educación contable, desarrollo normativos en contabilidad, tecnología contable, caracterización internacional de la contabilidad, nuevos desarrollos de la teoría contable, control interno, procesos de auditoría, revisoría fiscal (ver Machado, 2016). Lo cual fue complementado dentro de cada capítulo, con reflexiones que pueden orientar a los investigadores en torno a los aspectos de la metodología de la investigación que deberían considerar cuando van a iniciar la construcción de sus propuestas.

Seguramente existen muchos más temas con potencial de investigación y que algunos otros académicos del área contable ya vienen desarrollando, no obstante, se tendrá que evaluar detenidamente si los procesos de investigación de la institución van acordes a la coyuntura que hoy plantea la profesión contable, que presenta cambios rápidos y vertiginosos provenientes del impacto regulativo en el país o si por el contrario, se hacen apuestas por temáticas visionarias y de más largo plazo que tendrían cabida como nuevo conocimiento necesario en las organizaciones del futuro.

\section{UNA INVITACIÓN PARA CERRAR}

A pesar del desorden y las limitaciones que presenta la investigación contable en Colombia, se debe rescatar el interés permanente de hacer academia en el país, lo cual está en permanente crecimiento por la motivación de nuevos contadores públicos que se suman a la academia y que provienen principalmente, de procesos formativos en investigación, en los cuales se motivó hacia el camino de la generación de nuevo conocimiento para el avance de la contabilidad en el país.

Bajo este panorama, es necesario seguir fortaleciendo a los actuales y futuros investigadores contables del país en metodología de la investigación, pues las diversas miradas a los mismos problemas hacen que las alternativas de solución sean mucho más complejas y completas en aras del desarrollo general de la sociedad.

Por otro lado, se debe tener en cuenta que la nueva forma en que Publindex de COLCIENCIAS exigirá que las revistas colombianas que publiquen artículos contables, necesiten del denominado impacto por citación, para lo cual, se hace urgente que leamos a los colegas contables del país e iniciemos a reconocer sus trabajos de calidad y desconocer aquellos que efectivamente no se considera cumplen para la comunidad académica, pero que haga permanente el debate entre nosotros y con contables de otros países.

Para finalizar, se invita a la comunidad académica colombiana a escucharse, a debatirse, a reflexionarse, no solo internamente, sino también a la luz de lo que está sucediendo en el mundo para no caer en el eterno retorno. 


\section{REFERENCIAS BIBLIOGRÁFICAS}

Carvalho, J. A., Cadavid, L. A., Zapata, M. Á. y otros. (2006). Recreando el currículo. Medellín, Departamento de Ciencias Contables, Universidad de Antioquia.

Gómez, M. (2009). Los informes contables externos y la legitimidad organizacional con el entorno: estudio de un caso en Colombia. Innovar, 19(34), 147-166.

Grajales, J. S. (2010). La escritura de anteproyectos de investigación en un ambiente de no-lectores. El caso del curso metodología de la investigación contable en la Universidad del Valle Sede Norte del Cauca. Contaduría Universidad de Antioquia, 57, 145-159.

Grajales, J. S. (2007). Prejuicios hacia la contaduría pública: una mirada desde la ficción literaria. Contaduría Universidad de Antioquia, 51, 183-198.

Machado, M. A. (ed.). (2016). Caminos contables: problemas y metodologías para el desarrollo de la investigación. Medellín: Centro de investigaciones y consultorías, Facultad de Ciencias Económicas, Universidad de Antioquia.

Machado, M. A. (2012). Huellas y devenir contable: construyendo las rutas del pensamiento contable. Medellín: Centro de investigaciones y consultorías, Facultad de Ciencias Económicas, Universidad de Antioquia.

Machado, M. A. y Patiño, R. A. (2016). La investigación contable en Colombia. En, Machado (ed.) "Caminos contables: problemas y metodologías para el desarrollo de la investigación". Medellín: Centro de investigaciones y consultorías, Facultad de Ciencias Económicas, Universidad de Antioquia.

Macías, H.; Alzate, S.; Bautista, J. A. y otros. (2014). El futuro de las revistas contables colombianas: un debate polifónico. Cuadernos de contabilidad, 15 (37), 261-278.

Ospina, C.M. y Castaño, C.E. (2012). El campo de la teoría contable. Distinciones centrales para subrayar el papel de la Universidad de Antioquia en el contexto colombiano. En, M. Machado (ed.), "Huellas y devenir contable: construyendo las rutas del pensamiento contable" (pp. 222-275). Medellín: Centro de investigaciones y consultorías, Facultad de ciencias económicas, Universidad de Antioquia.

Rojas, W. (2009). Congoja por una educación contable fútil. En Archel y otros, "Irrupciones significativas para pensar la contabilidad". Cali: Facultad de ciencias de la administración, Universidad del Valle.

Rueda, G. (2009). Editorial. Orígenes de la producción académica contable. Cuadernos de contabilidad, 10(26), 9-11.

Rueda, G. (2007). La investigación contable: vínculos ontológicos y las posibilidades de la investigación interpretativa. Contaduría Universidad de Antioquia, 50, 119-132.

Ryan, B., Scapens, R., y Theobald, M. (2004). Metodología de la investigación en finanzas y contabilidad. Barcelona: Ediciones Deusto.

Castaño-Rios, C. (2016). Reflexiones sobre la investigación contable en Colombia. Panorama Económico, 24, 163-174.

\section{AUTORES}

Carlos Eduardo Castaño-Rios

Magíster en administración y contador público de la Universidad de Antioquia. Profesorinvestigador del Departamento de Ciencias Contables y actual Director del Centro de Investigaciones y Consultorías -CIC- de la Facultad de Ciencias Económicas de la Universidad de Antioquia. Secretario técnico de la red colombiana de editores y revistas de contabilidad - REDITORES. 